\title{
Hydrogen Tuning of (InGa)(AsN) Optical Properties
}

\author{
G. Baldassarri H. v. H., F. Ranalli, M. Bissiri, \\ V. Gaspari, A. Polimeni, M. Capizzi, A. Nucara
}

INFM-Dipartimento di Fisica, Università degli Studi di Roma "La Sapienza"

Piazzale A. Moro 2, 00185 Roma, Italy

\section{GEDDO}

INFM-Dipartimento di Fisica, Università di Parma

43010 Fontanini (Parma), Italy

\section{Fischer, M. Reinhardt and A. Forchel}

Universität Würzburg, Technische Physik

Am Hublaund, 97074 Würzburg, Germany

The effects of atomic hydrogen irradiation on the optical properties of $(\mathrm{InGa})(\mathrm{AsN})$ single quantum wells investigated by means of photoluminescence spectroscopy. For increasing hydrogen dose, the photoluminescence band peak energy of each nitrogen-containing sample blue-shifts and for high hydrogen dose it reaches that of a corresponding nitrogen-free reference sample. This effect is accompanied by a broadening of the photoluminescence band line width and by a decrease in the photoluminescence efficiency. Thermal annealing at $550^{\circ} \mathrm{C}$ fully restores the original band gap value and the photoluminescence line width of the sample before hydrogenation. An interpretation of these phenomena is proposed in terms of an $\mathrm{H}$ perturbation of the charge distribution around the strongly electronegative $\mathrm{N}$ atoms, leading most likely to the formation of $\mathrm{H}-\mathrm{N}$ complexes, and to an ensuing electronic passivation of nitrogen.

PACS numbers: 78.66.Fd, 71.55.Eq, 78.55.Cr

Among all the atoms, hydrogen is known to be the smallest and one of the most reactive. Moreover, it is highly diffusive when introduced in solids. These features make $\mathrm{H}$ a useful tool for passivating defects in semiconductors, such as deep centers or dislocations, and justify the huge scientific interest in its effects 
in $\mathrm{Si}, \mathrm{GaAs}$ or GaN [1]. From this point of view, it is interesting to study the role of hydrogen in III-N-V alloys such as InGaAsN, a material of relevance for telecommunications via optical fibers [2]. The insertion of a few nitrogen percent in (InGa)As leads to a giant, composition dependent band gap bowing [3] and to an increase in the electron effective mass [4], which have been ascribed to the strongly localized nature of the perturbation induced by nitrogen in the host lattice and explained in terms of point symmetry-breaking arguments $[5,6]$ or band anticrossing between N-related levels and conduction band states $[7,8]$.

In this paper, we show that $\mathrm{H}$ insertion in (InGa)(AsN) quantum wells (QWs), or its subsequent removal, allow a post-growth tuning of the optical properties of the samples. In particular, for increasing $\mathrm{H}$ dose the band gap of the $\mathrm{N}$-containing quantum wells blueshifts toward that of the corresponding $\mathrm{N}$-free reference samples (or blanks), where it saturates at the highest $\mathrm{H}$ doses. Thermal annealing at $550^{\circ} \mathrm{C}$ fully recovers the optical properties the samples had before hydrogenation. An interpretation of these findings is here proposed in terms of the formation of $\mathrm{H}-\mathrm{N}$ bonds, resulting in a decrease in the effective $\mathrm{N}$ content and a subsequent band gap re-opening.

The investigated $\operatorname{In}_{x} \mathrm{Ga}_{1-x} \mathrm{As}_{1-y} \mathrm{~N}_{y}$ single QWs were grown by solid source molecular beam epitaxy. Indium, $x$, and nitrogen, $y$, concentrations range from 0.25 to 0.41 and from 0.007 to 0.052 , respectively, and well width, $L$, from 6 to $8 \mathrm{~nm}$. All samples have a $100 \mathrm{~nm}$ thick GaAs capping layer. Post-growth treatment with atomic hydrogen irradiation at $300^{\circ} \mathrm{C}$ was supplied by a Kaufman source, with an ion energy of $100 \mathrm{eV}$, a current density of few tens of $\mu \mathrm{A} / \mathrm{cm}^{2}$ and hydrogen doses, $d_{\mathrm{H}}$, from 1 to $690 H_{0}\left(H_{0}=1.0 \times 10^{16}\right.$ ions $\left.\mathrm{cm}^{-2}\right)$.

One-hour thermal annealing was performed at $10^{-6}$ Torr and various temperatures between $220^{\circ} \mathrm{C}$ and $550^{\circ} \mathrm{C}$. Photoluminescence (PL), excited by the $515 \mathrm{~nm}$ line of an $\mathrm{Ar}^{+}$laser, was dispersed by a single $1 \mathrm{~m}$ monochromator and detected by a Ge detector cooled at liquid nitrogen temperature. Here we focus on the results obtained in the QW with the highest $\mathrm{N}$ content $(y=0.052), x=0.38$ and $L=8 \mathrm{~nm}$ and in its corresponding blank

Figure 1 shows the PL spectra at $150 \mathrm{~K}$ of the N-containing sample and of its corresponding reference blank. For increasing $\mathrm{H}$ dose (from bottom to top), the PL peak of the $y=0.052 \mathrm{QW}$ blueshifts toward that of the blank (dashed line), which remains almost fixed in energy. At the highest $\mathrm{H}$ dose, the peak energy of the hydrogenated sample overlaps that of the N-free QW. Hydrogen irradiation also leads to an increased microscopic disorder, as witnessed by the broadening of the PL band line width. Finally, PL intensity steadily decreases in the $y=0.052 \mathrm{QW}$, and increases by a factor of 5 in the blank for $d_{\mathrm{H}}=H_{0}$, before dropping at the highest $d_{\mathrm{H}}$ (not shown here). The effects of hydrogen irradiation can be reversed by thermal annealing. Figure 2 shows the PL spectra of the N-containing QW (hydrogenated at $d_{\mathrm{H}}=100 H_{0}$ ) for different annealing temperatures, $T_{\mathrm{a}}$. For increasing $T_{\mathrm{a}}$ (from bottom to top), the PL band of the $y=0.052 \mathrm{QW}$ moves 


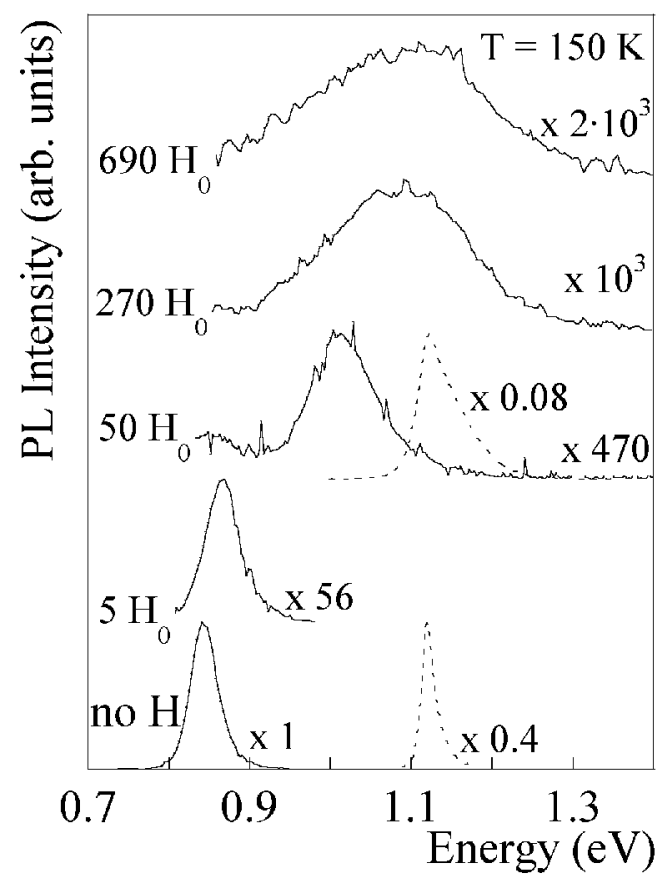

Fig. 1. Normalized photoluminescence spectra at $T=150 \mathrm{~K}$ of two $\mathrm{In}_{0.38} \mathrm{Ga}_{0.62} \mathrm{As}_{1-y} \mathrm{~N}_{y} / \mathrm{GaAs}$ QWs with $y=0.052$ (continuous line) and $y=0.0$ (dashed line), hydrogenated at different $H$ doses $\left(H_{0}=1.0 \times 10^{16}\right.$ ions $\left./ \mathrm{cm}^{2}\right)$. Normalization factors are given for each spectrum. Laser power density $P=300 \mathrm{~W} / \mathrm{cm}^{2}$.

toward lower energy, until its line shape nearly coincides with that of the $\mathrm{H}$-free, not annealed sample. These results are summarized in Fig. 3, where the half-width at half maximum, HWHM, of the PL band of the $\operatorname{In}_{0.38} \mathrm{Ga}_{0.62} \mathrm{As}_{0.948} \mathrm{~N}_{0.052} \mathrm{QW}$ is reported versus its PL peak energy, $E_{\mathrm{p}}$, for $\mathrm{H}$ doses increasing from 0 to $250 H_{0}$ (full squares, upward arrow) and for annealing temperatures increasing from 200 to $600^{\circ} \mathrm{C}$ in the sample with $d_{\mathrm{H}}=100 H_{0}$ (open circles, downward arrow). HWHM has been evaluated from the low energy side in order to avoid spurious contributions to the PL line width due to band filling effects.

This behavior establishes quite a reversible cycle, showing hydrogen to be a useful tool for tailoring the optical properties of (InGa)(AsN) QWs. We now discuss in some detail the possible microscopic origin of this behavior. When introduced in a solid, the charge state of hydrogen is determined by the Fermi level of the host lattice and hydrogen behaves as an amphoteric impurity. Our samples are unintentionally $p$-doped due to residual $\mathrm{C}$ in the MBE chamber, so that hydrogen enters in the lattice in a positively charged state, $\mathrm{H}^{+}$. On the other hand, due to its strong electronegativity with respect to $\mathrm{Ga}$ and As atoms, nitrogen leads to an accumulation of negative charge in its surroundings. This condition favors the 


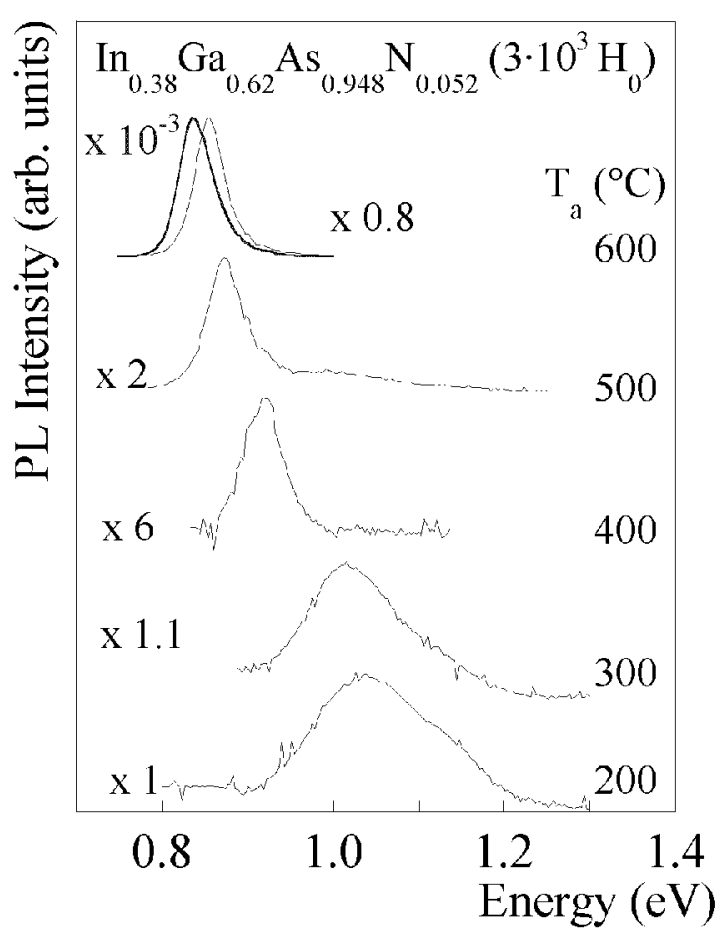

Fig. 2. Normalized photoluminescence spectra at $T=150 \mathrm{~K}$ of a $d_{\mathrm{H}}=100 H_{0}$ $\mathrm{In}_{0.38} \mathrm{Ga}_{0.62} \mathrm{As}_{0.948} \mathrm{~N}_{0.052} / \mathrm{GaAs} \mathrm{QW}$ (continuous line) for different annealing temperatures, $T_{\mathrm{a}}$. The photoluminescence spectrum of the $\mathrm{H}$-free, not annealed $\mathrm{QW}$ is reported for comparison dotted line). Normalization factors are given for each spectrum. Laser power density $P=900 \mathrm{~W} / \mathrm{cm}^{2}$.

formation of $\mathrm{H}^{+}-\mathrm{N}^{-}$bonds, in agreement with theoretical proposals and experimental suggestions in p-type GaN [9]. The resulting "molecular orbital" between $\mathrm{H}^{+}$and $\mathrm{N}^{-}$may lead to a bonding level deep in the gap, with a subsequent $\mathrm{N}$ passivation and a decrease in the effective $\mathrm{N}$ content. Thermal annealing breaks this bond, increasing back the $\mathrm{N}$ content in the well. We stress that no change in the PL peak energy of the blank and of a H-free $y=0.052$ was observed upon annealing.

Hydrogen affects also PL band intensity and line shape. The behavior of the PL intensity of the $y=0.052$ QW in Fig. 1 can be interpreted in terms of two competing processes: the passivation of preexisting defects (as it happens in the blank) and the introduction of new nonradiative centers upon hydrogenation. Finally, the quenching of the PL efficiency at high hydrogen doses, or the absence of a complete recover of the PL intensity at high annealing temperature, can be ascribed to bombardment damage and As desorption, respectively.

As far as the PL line shape is concerned, the HWHM of the hydrogenated 


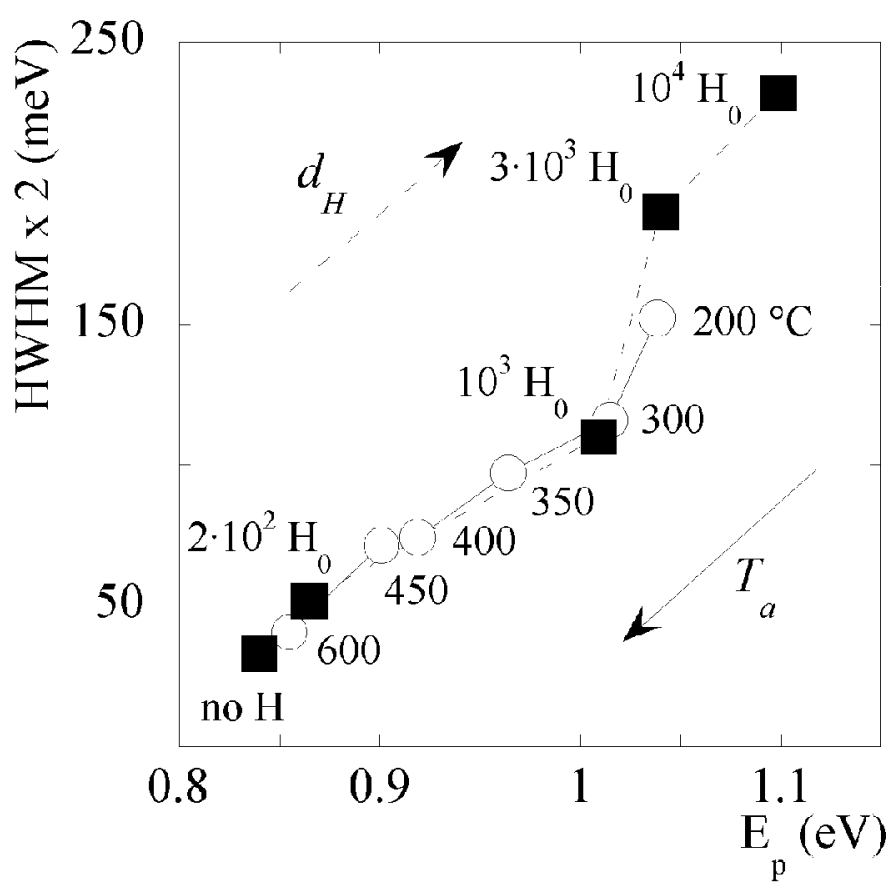

Fig. 3. Half-width at half maximum, of the photoluminescence band in $\mathrm{In}_{0.38} \mathrm{Ga}_{0.62} \mathrm{As}_{0.948} \mathrm{~N}_{0.052} / \mathrm{GaAs} \mathrm{QW}$ versus the PL band peak energy, $E_{\mathrm{p}}$. Data are taken from $T=150 \mathrm{~K}$ photoluminescence spectra. Full squares refer to a $\mathrm{QW}$ hydrogenated at increasing (upward arrow) H doses, $d_{\mathrm{H}}$, open circles refer to a QW hydrogenated at $d_{\mathrm{H}}=100 H_{0}\left(=10^{18}\right.$ ions $\left.\mathrm{cm}^{-2}\right)$ and annealed at increasing (downward arrow) temperatures, $T_{\mathrm{a}}$.

$\mathrm{In}_{x} \mathrm{Ga}_{1-x} \mathrm{As}_{1-y} \mathrm{~N}_{y}$ QWs increases with $E_{\mathrm{p}}$, because more hydrogen (irradiated or residual) leads to a higher $\mathrm{N}$ passivation, but also to an increased microscopic disorder due to local fluctuations of the effective $\mathrm{N}$ concentration in the well and/or to different types of $\mathrm{N}-\mathrm{H}$ bonds.

In conclusion, hydrogen demonstrates to be a powerful tool for investigating the electronic properties of $\mathrm{N}$ in $(\mathrm{InGa})(\mathrm{AsN}) \mathrm{QWs}$. Present results show a strongly localised interaction between $\mathrm{N}$ and $\mathrm{H}$. In turn, this interaction leads to an effective lowering of the $\mathrm{N}$ content and to a band gap blue-shift of the ( $\mathrm{InGa}$ (AsN) material. This suggests an "impurity-like" character of $\mathrm{N}$ in $(\mathrm{InGa})(\mathrm{AsN})$ even in the "alloy" limit.

\section{References}

[1] Hydrogen in Semiconductors, Eds. J.I. Pankove, N.M. Johnson, Semiconductors and Semimetals, Vol. 34, Academic Press, New York 1991. 
[2] M. Kondow, K. Uomi, A. Niwa, T. Kitatani, S. Watahiki, Y. Yazawa, Jpn. J. Appl. Phys. 35, 1273 (1996).

[3] P.R.C. Kent, A. Zunger, Phys. Rev. Lett. 86, 2609 (2001).

[4] P.N. Hai, W.M. Chen, I. Buyanova, X.P. Xin, C.W. Tu, Appl. Phys. Lett. 77, $1843(2000)$.

[5] T. Mattila, S.H. Wei, A. Zunger, Phys. Rev. B 60, R11245 (1999).

[6] E.D. Jones, N.A. Modine, A.A. Allerman, S.R. Kurtz, A.F. Wright, S.T. Tozer, X. Wei, Phys. Rev. B 60, 4430 (1999).

[7] W. Shan, W. Walukiewicz, J.W. Ager III, E.E. Aller, J.F. Geisz, D.J. Friedman, J.M. Olson, S.R. Kurtz, Phys. Rev. Lett. 82, 1221 (1999).

[8] A. Lindsay, E.P. O'Reilly, Solid State Commun. 112, 443 (1999).

[9] J. Neugebauer, C. Van de Walle, Phys. Rev. Lett. 75, 4452 (1995). 